\title{
On the apsidal motion of MY Cygni (Research Note)
}

\author{
M. Wolf \\ Astronomical Institute, Faculty of Mathematics and Physics, Charles University Prague, 18000 Praha 8, V Holešovičkách 2, \\ Czech Republic \\ e-mail: wolf@cesnet.cz
}

Received 17 February 2009 / Accepted 24 February 2009

\section{ABSTRACT}

\begin{abstract}
MY Cygni is a relatively bright eclipsing binary system with a slightly eccentric orbit. Tucker and collaborators found that it shows retrograde apsidal motion in contradiction with theory. Based on a new $\mathrm{O}-\mathrm{C}$ diagram solution, we concluded that the apsidal motion is prograde in agreement with theory, and its period is about 1400 years. The determined internal structure constant is close to the theoretically expected value. The relativistic effect is significant, being about $18 \%$ of the total apsidal motion rate.
\end{abstract}

Key words. stars: binaries: eclipsing - stars: individual: MY Cyg - stars: fundamental parameters

\section{Introduction}

The eclipsing nature of the 8th magnitude star MY Cygni (HD 193637, BD $+33^{\circ} 3862$, HIP 100258, Sp. A7m) was discovered by Hoffmeister (1930) on plates of Sonneberg Observatory. The first photographic light curves were presented by Wachmann (1948) and Gaposchkin (1953) with the period of 2 days. The correct 4-day orbital period was confirmed later from spectroscopy by Popper (1969). The system has a slightly eccentric orbit $(e \simeq 0.01)$. With its period of almost precisely an integer number of days, MY Cyg is a difficult object to study with light curve photometry.

Tucker et al. (2009, hereafter T09) derived orbital solutions and precise absolute dimensions of the similar components (Table 3). They also established an apsidal motion period $(U \simeq 1700 \mathrm{yr})$ and found a retrograde/negative value of the apsidal motion rate ( $\dot{\omega}=-0.21 \pm 0.07 \mathrm{deg} / \mathrm{year})$. If MY Cyg does have a retrograde apsidal motion, it would be a new candidate that contradicts theory and requires further study.

The historical O-C diagram for MY Cyg was presented in Kreiner et al. (2001). This system is also included in the current compilation of eclipsing binaries with eccentric orbits (Bulut \& Demircan 2007) as well as in the Catalogue of close binaries in $\delta$ Scuti region (Soydugan et al. 2006).

\section{Apsidal motion}

MY Cyg has a small but significant orbital eccentricity. Both the classical theory of tides and General Relativity predict that such a close system should exhibit a periastron advance. We completed a new apsidal motion study of MY Cyg by performing an $\mathrm{O}-\mathrm{C}$ diagram analysis and adopting a complete list of times of minimum light. The method described by Giménez \& Bastero (1995), which is a weighted least-squares iterative procedure, including terms in the eccentricity up to the fifth order, was routinely used. The periastron position $\omega$ at epoch $E$ is defined by the linear equation

$\omega=\omega_{0}+\dot{\omega} E$

where $\dot{\omega}$ is the rate of periastron advance, and the position of periastron for the zero epoch $T_{0}$ is denoted as $\omega_{0}$. The relation between the sidereal and the anomalistic period, $P_{\mathrm{s}}$ and $P_{\mathrm{a}}$, is given by

$P_{\mathrm{s}}=P_{\mathrm{a}}\left(1-\dot{\omega} / 360^{\circ}\right)$,

and the period of apsidal motion by

$U=360^{\circ} P_{\mathrm{a}} / \dot{\omega}$

Numerous eclipse timings for MY Cyg have been reported in the literature using a variety of techniques. Photoelectric or CCD measurements have greater precision and are the most useful for our analysis. The additional CCD photometry of MY Cyg was obtained by Ms. Kučáková on May 7, 2008. The observations were carried out with the $20-\mathrm{cm}$ reflecting telescope of the Johann Palisa Observatory and Planetarium, which is located at Ostrava, Czech Republic. The SBIG ST-8 CCD camera and Johnson's VRI filters were used. The C-MUNIPACK ${ }^{1}$ (Motl 2007), the user-friendly software package, was used for reduction of our CCD images. Using the Hipparcos photometry (ESA 1997), we were able to derive three additional secondary times of minimum light with lower accuracy. The light-curve profile fitting method was used. The epochs in Table 1 were computed according to the linear ephemeris of T09:

Pri. Min. = HJD $2441585.62896+4.005187081 \cdot E$, Sec. Min. = HJD $2441587.64061+4.005188234 \cdot E$.

\footnotetext{
${ }^{1}$ http://c-munipack. sourceforge.net/
} 
Table 1. New times of minimum light.

\begin{tabular}{llccl}
\hline \hline $\begin{array}{l}\text { JD Hel.- } \\
2400000\end{array}$ & $\begin{array}{l}\text { Error } \\
\text { [day] }\end{array}$ & Epoch & $\begin{array}{c}\text { Method } \\
\text { Filter }\end{array}$ & $\begin{array}{l}\text { Observatory } \\
\text { Source }\end{array}$ \\
\hline 47859.766 & 0.002 & 1566.5 & pe, H & Hipparcos \\
48104.081 & 0.002 & 1627.5 & pe, H & Hipparcos \\
48500.596 & 0.002 & 1726.5 & pe, H & Hipparcos \\
$54594.47660^{*}$ & 0.0004 & 3248.0 & CCD, R & Ostrava \\
$54594.47762^{*}$ & 0.0006 & 3248.0 & CCD, V & Ostrava \\
$54594.47773^{*}$ & 0.0014 & 3248.0 & CCD, I & Ostrava \\
\hline
\end{tabular}

Note: * presented also in Brát et al. (2008).

Table 2. Apsidal motion elements of MY Cyg.

\begin{tabular}{|c|c|c|c|}
\hline Parameter & $\overline{\text { Unit }}$ & $\overline{\text { T09 }}$ & This paper \\
\hline$T_{0}$ & HJD & - & $2441585.6334(3)$ \\
\hline$P_{\mathrm{s}}$ & days & 4.005186427 & $4.00518779(12)$ \\
\hline$P_{\mathrm{a}}$ & days & - & $4.00521882(12)$ \\
\hline$e$ & - & $0.010(1)$ & $0.0094(2)$ \\
\hline$\dot{\omega}$ & deg cycle $^{-1}$ & - & $0.0028(3)$ \\
\hline$\dot{\omega}$ & $\operatorname{deg} \mathrm{yr}^{-1}$ & $-0.21(7)$ & $0.25(3)$ \\
\hline$\omega_{0}$ & deg & $69.6(2.4)$ & $291.7(0.4)$ \\
\hline$U$ & years & 1700 & $1420(160)$ \\
\hline
\end{tabular}

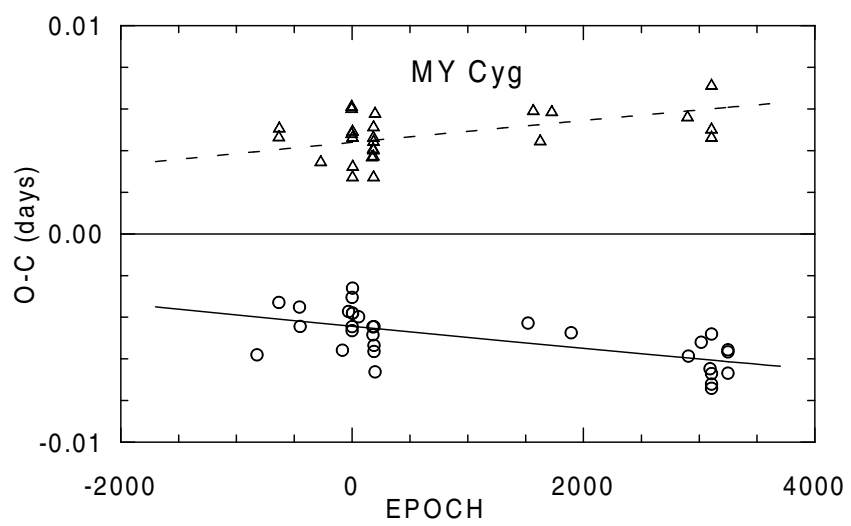

Fig. 1. The O-C diagram for the times of minimum of MY Cyg. The continuous and dashed lines represent predictions for the primary and secondary eclipses, respectively. The individual primary and secondary minima are denoted by circles and triangles, respectively.

Besides those minima given in Table 1, we added the photoelectric times of minimum obtained by Pohl \& Kizilirmak (1964, 1966, 1975), Williamon (1975, his Table V), Tremko et al. (1979, their Table 2), Blättler (1993), Caton \& Smith (2005), Hübscher et al. (2006), and T09 (their Table 8). A total of 57 reliable photoelectric times of minimum light were used in our dataset, 26 secondary eclipses being among them. The orbital inclination was assumed to be $i=88^{\circ} .58$, based on the current photometric analysis (T09). The computed apsidal motion parameters and the internal errors of the least-squares fit are given in Table 2, and the $\mathrm{O}-\mathrm{C}$ diagram is shown in Fig. 1.

\section{Internal structure constant}

Observations of eccentric binary systems with apsidal motion allow us to determine the internal structure constant, $k_{2}$, which is an important parameter in stellar evolution models. The observed average value of $\bar{k}_{2 \text {,obs }}$ is given by

$\bar{k}_{2, \mathrm{obs}}=\frac{1}{c_{21}+c_{22}} \frac{P_{\mathrm{a}}}{U}=\frac{1}{c_{21}+c_{22}} \frac{\dot{\omega}}{360}$,
Table 3. Astrophysical parameters of MY Cyg.

\begin{tabular}{lcc}
\hline \hline Parameter & Unit & Value \\
\hline$M_{1}$ & $M_{\odot}$ & $1.82(3)$ \\
$M_{2}$ & $M_{\odot}$ & $1.80(3)$ \\
$r_{1}$ & - & $0.1359(6)$ \\
$r_{2}$ & - & $0.1400(6)$ \\
$i$ & deg & $88.58(2)$ \\
\hline$\dot{\omega}_{\text {rel }}$ & deg cycle & 0.00051 \\
$\dot{\omega}_{\text {rel }} / \dot{\omega}$ & $\%$ & 18.3 \\
$\log k_{2, \text { obs }}$ & - & $-2.43(3)$ \\
$\log k_{2, \text { theo }}$ & - & -2.45 \\
\hline
\end{tabular}

where $c_{21}$ and $c_{22}$ are well-known functions of the orbital eccentricity, fractional radii, the masses of the components, and the ratio of the rotational velocity of the stars to the Keplerian velocity (Kopal 1978). The rotation of the stars was assumed to be synchronized with the maximum angular orbital velocity achieved at periastron (pseudo-synchronous rotation). Taking the value of the eccentricity and the masses of the components into account, one has to subtract a relativistic correction $\dot{\omega}_{\text {rel }}$ (Giménez 1985)

$\dot{\omega}_{\text {rel }}=5.45 \times 10^{-4} \frac{1}{1-e^{2}}\left(\frac{M_{1}+M_{2}}{P}\right)^{2 / 3}$,

where $M_{i}$ denotes the individual masses of the components in solar units and $P$ is the orbital period in days. The values of $\dot{\omega}_{\text {rel }}$ and the resulting mean internal structure constant $\bar{k}_{2, \text { obs }}$ are given in Table 3. Its error was evaluated according to the relation presented in Wolf \& Zejda (2005), and the uncertainity in the eccentricity was neglected due to the presence of its higher terms. The theoretical value $k_{2 \text {,theo }}$ was taken as a mean value from the tables of the Granada stellar models according to the adopted masses and chemical composition (Claret 2004). The chemical composition of $(X, Z)=(0.70,0.02)$ and $(0.70,0.03)$ was supposed to agree with previous studies.

\section{Summary}

MY Cygni is an eccentric eclipsing binary exhibits slow apsidal motion. Our new $\mathrm{O}-\mathrm{C}$ diagram analysis has proved that the apsidal motion is prograde and has a period of about 1400 years. The prograde motion means that MY Cyg is not a problematic case, and is in agreement with theory. We concluded that the negative apsidal motion rate determined by T09 was due to their relatively short observational time interval and the method used for its determination.

Nevertheless, the radial velocity amplitudes $K_{1}$ and $K_{2}$ presented in T09 have quite large uncertainties compared to those given by Popper (1971). Moreover, they are not in agreement with the given absolute dimensions, cf. their Tables 4 and 5. It is also clearly evident that our resulting $\omega_{0}=2911^{\circ} 7$ is close to the value $2 \pi-\omega_{0}$ given in T09.

We also calculated the internal structure constant of MY Cyg and found that it is close to its theoretical value. The slight difference should be removed in the future with the compilation of a more comprehensive $\mathrm{O}-\mathrm{C}$ diagram. Therefore, minima observations of MY Cygni are needed in the future.

Acknowledgements. This research was supported by the Research Program MSM0021620860 of the Ministry of Education of the Czech Republic and has made use of the SIMBAD database, operated at CDS, Strasbourg, France, and 
of NASA's Astrophysics Data System Bibliographic Services. We gratefully acknowledge useful suggestions by the referee, Prof. J. V. Clausen.

\section{References}

Blättler, E. 1993, BBSAG Bull., 104

Brát, L., Šmelcer, L., Kučáková, H., et al. 2008, OEJV, 94, 1

Bulut, I., \& Demircan, O. 2007, MNRAS, 378, 179

Caton, D. B., \& Smith, A. B. 2005, IBVS, 5595

Claret, A. 2004, A\&A, 424, 919

ESA 1997, The Hipparcos and Tycho Catalogues, ESA SP-1200

Gaposchin, S. 1953, Harvard Ann., 113, 105

Giménez, A. 1985, ApJ, 297, 405

Giménez, A., \& Bastero, M. 1995, Ap\&SS, 226, 99

Hoffmeister, C. 1930, AN, 240, 195

Hübscher, J., Paschke, A., \& Walter, F. 2006, IBVS, 5731

Kopal, Z. 1978, Dynamics of Close Binary Systems (Dordrecht, Holland: Reidel)
Kreiner, J. M., Kim, C.-H., \& Nha, I.-S. 2001, An Atlas of O-C diagrams of Eclipsing Binary Stars, Wydawnictwo Naukowe Akademii Pedagogicznej, Cracow, Poland

Motl, D. 2007, C-MunIPACK, http: //c-munipack . sourceforge.net/

Pohl, E., \& Kizilirmak, A. 1964, AN, 288, 69

Pohl, E., \& Kizilirmak, A. 1966, AN, 289, 191

Pohl, E., \& Kizilirmak, A. 1975, IBVS, 1053

Popper, D. M. 1969, BAAS, 1, 257

Popper, D. M. 1971, ApJ, 169, 549

Soydugan, E., Soydugan, F., Demircan, O., \& Ibanoglu, C. 2006, MNRAS, 370, 2013

Tremko, J., Papoušek, J., \& Vetešník, M. 1979, CoSka, 8, 159

Tucker, R. S., Sowell, J. R., Williamon, R. M., \& Coughlin, J. L. 2009, AJ, 137, 2949

Wachmann, A. A. 1948, Astron. Abhandlungen Hamburg, 11, 40

Williamon, R. M. 1975, AJ, 80, 976

Wolf, M., \& Zejda, M. 2005, A\&A, 437, 545 